\title{
Effects of Water on Seismic Wave Velocities in the Upper Mantle
}

\author{
By Shun-ichiro KARATO \\ Department of Geology and Geophysics, University of Minnesota, Minneapolis, MN 55455 \\ (Communicated by Yoshibumi Tomoda, M. J. A., Feb. 13, 1995)
}

\begin{abstract}
Possible roles of water to affect seismic wave velocities in the upper mantle of the Earth are examined based on mineral physics observations. Three mechanisms are considered: (i) direct effects through the change in bond strength due to the presence of water, (ii) effects due to the enhanced anelastic relaxation and (iii) effects due to the change in preferred orientation of minerals. It is concluded that the first direct mechanism yields negligibly small effects for a reasonable range of water content in the upper mantle, but the latter two indirect effects involving the motion of crystalline defects can be significant. The enhanced anelastic relaxation will significantly (several \%) reduce the seismic wave velocities. Based on the laboratory observations on dislocation mobility in olivine, a possible change in the dominant slip direction in olivine from [100] to [001] and a resultant change in seismic anisotropy is suggested at high water fugacities. Changes in seismic wave velocities due to water will be important, particularly in the wedge mantle above subducting oceanic lithosphere where water content is likely to be large.
\end{abstract}

Key words : Water; seismic wave velocity; upper mantle; anelasticity; seismic anisotropy.

Introduction. Water plays important roles in the melting processes and hence resultant chemical evolution of the solid Earth. ${ }^{1)}$ Water is also known to affect the transport properties of silicate minerals including diffusion of atoms ${ }^{2)}$ and creep rate. ${ }^{3), 4)}$ Thus understanding of how water is circulated and distributed in the Earth's mantle is a key issue in the study of evolution and dynamics of the Earth's interior. One way to infer the distribution of water in the mantle is to analyze samples from the Earth's interior. ${ }^{5)}$ However, this technique is limited by the availability of specimens. An alternative method is to use some geophysical observations to infer the water distribution. For instance, Karato ${ }^{6)}$ suggested that the presence of water will increase electrical conductivity of olivine. Thus the measurements of electrical conductivity by geomagnetic sounding methods ${ }^{7)}$ could provide constraints on water content if the relation between water content and conductivity is known. Unfortunately, however, estimation of electrical conductivities in the Earth can be made only with large uncertainties, and the results are not very conclusive. ${ }^{7)}$

Velocities of seismic waves are among the best constrained physical parameters of the Earth's in- terior. Therefore, if one understands the relation between water content and seismic wave velocities, one will get a powerful tool to infer the water content in the Earth. To my knowledge, however, no experimental studies have been performed to examine the effects of water on seismic wave velocities in minerals. Nevertheless, existing mineral physics observations on the solubility of water ${ }^{8), 9)}$ and its role on defect mobilities in upper mantle minerals ${ }^{3,4), 10), 11)}$ allow us to formulate some working hypotheses about the effects of water on seismic wave velocities, which are to be tested by future experimentation.

Throughout this paper, I will assume that water is present as solid solution in major constituent minerals especially olivine. Recent laboratory data by Kohlstedt and his coworkers ${ }^{8), 9)}$ demonstrated that olivine can dissolve a significant amount of water under upper mantle conditions, hence most of the water (up to $0.1 \mathrm{wt} \%$ : e.g. ${ }^{1)}$ ) can be incorporated in olivine rather than in some hydrous phases. It is the effects of this type of water that is investigated in this paper. Similar models may also apply for other minerals but not much data are available for them to make any educated inferences.

Mechanisms of water effects on seismic wave 
velocities. (i) Direct effect through the change in bond strength. The most direct mechanism is the effect due to the change in bond strength. When water (hydrogen) is dissolved in silicates, some chemical bonds will be replaced with weaker bonds such as H.O. Seismic wave velocity (V) is related to the bond strength (elastic constant, c) through,

$$
V=\sqrt{c / \rho}
$$

where $\rho$ is the density. Both density and elastic constant will be reduced by the incorporation of water. When the amount of water ( $\phi$ : volume fraction) is small, the effects of water can be written as

$$
\delta V / V=-A \phi
$$

where $A$ is a constant which depends on the mechanisms of softening and elastic constants. To get a maximum effect, I assume that the chemical bonds related to water (such as $\mathrm{O}-\mathrm{H}$ ) are much weaker than other bonds (e.g., Si-O, Mg-O) and therefore the effects of water is estimated using a model for elastic properties of materials containing spherical pores. ${ }^{12)}$ Asssuming $v$ (Poisson's ratio) $\sim 0.25$, I get $\hat{A}_{\mathrm{P}}=0.54$ and $\mathrm{A}_{\mathrm{S}}=0.49$ where $\mathrm{A}_{\mathrm{P}}$ and $\mathrm{A}_{\mathrm{S}}$ are constants in equation $^{2)}$ for $\mathrm{P}$ - and S-waves respectively. Therefore, for $\phi=10^{-2}-10^{-3}(1,5), \delta V / V \sim 0.5 \times\left(10^{-2}-10^{-3}\right)$, which is negligibly small.

(ii) Indirect effects through the enhancement of anelastic relaxation. Effects of anelastic relaxation on seismic wave velocities are large particularly in the asthenosphere where $Q\left(Q^{-1}\right.$ is the energy dissipation of seismic waves) is low. ${ }^{13)}$ The reduction of seismic wave velocity due to anelastic relaxation is given by, ${ }^{13)}$

$$
\delta V / V=-(1 / 2) \cot (\pi \alpha / 2) Q^{-1}(\omega)
$$

where $\delta V$ is the velocity reduction due to anelasticity, $\alpha$ a parameter to describe the frequency $(\omega)$ dependence of energy dissipation $\left(Q^{-1}\right)$, viz.,

$$
Q^{-1}(\omega) \sim(\omega \tau)^{-\alpha}
$$

where $\tau$ is the relaxation time of anelasticity. Seismological and laboratory observations indicate $\alpha=0.1-0.3{ }^{14)}$ For this range of $\alpha, \delta V / V \sim-(1-6) \%$ for the $Q$ of 50 to 100 . Thus a change in $Q$ will result in a significant change in seismic wave velocities. The relaxation time is related to the mobility $(B)$ of relevant defects that are responsible for anelastic relaxation,

$$
\tau \sim B^{-1} .
$$

Therefore, one expects a smaller $Q$ and more significant reduction of seismic velocities, when the mobility is enhanced.

Laboratory data show that the mobilities of defects in olivine including point defects, dislocations and grain-boundaries are enhanced by the presence of a small amount of water. ${ }^{3), 4), 10), 11)}$ Combining equations [3], [4] and [5], one finds,

$$
\frac{(\delta V / V)_{\mathrm{wet}}}{(\delta V / V)_{\mathrm{dry}}}=\frac{Q_{\mathrm{wet}}^{-1}}{Q_{\mathrm{dry}}^{-1}}=\left(\frac{B_{\mathrm{wet}}}{B_{\mathrm{dry}}}\right)^{\alpha}
$$

where $X_{\text {wet,dry }}\left(X=\delta V / V, Q^{-1}, B\right)$ indicates $X$ under wet (water-rich) or for dry (water-poor) conditions respectively. Fig. 1 shows the effects of water on seismic wave velocities through the enhancement of defect mobility. It is found that the effect is significant for $B_{\text {wet }} / B_{\text {dry }}>10$. The laboratory data indicate $B_{\text {wet }} / B_{\text {dry }} \sim 10-10^{2}$ for the range of conditions so far explored (namely at pressures less than 0.3 GPa). Considering the fact that the solubility of water in olivine at higher pressures is significantly higher than those at these conditions, ${ }^{9)}$ I conclude that the effects of water to reduce seismic wave velocity can be large in the upper mantle, when the water fugacity is high.

(iii) Effects of water on seismic anisotropy. Significant seismic anisotropy has been detected in the upper mantle of the Earth. ${ }^{15), 16)}$ Mineral physics interpretation of seismic anisotropy usually assumes that the preferred orientation of olivine is responsible for anisotropy and further that the preferred orientation of minerals is similar to that found in the uppermost oceanic mantle (ophiolites) which are deformed under relatively dry conditions. ${ }^{16), 17)}$ Under these conditions, the dominant slip system in olivine is $[100](010)$ and hence the olivine [100] axes become subparallel to the macroscopic flow direction and olivine (010) plane becomes subparallel to the macroscopic flow plane. This results in the seismic anisotropy such that the direction of the fast $\mathrm{P}$-wave and the polarization of the fast S-wave are subparallel to the flow direction. ${ }^{18)}$

However, existing data on the role of water on olivine deformation suggest that the dominant slip systems might change under high water fugacities. Fig. 2 shows the effects of water on the creep strength 
(A) velocity reduction due to anelasticity

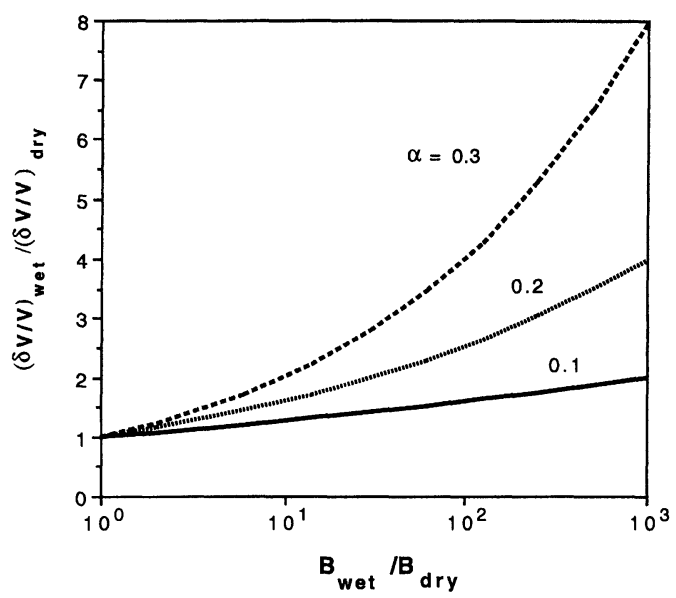

(B)

velocity reduction due to anelasticity

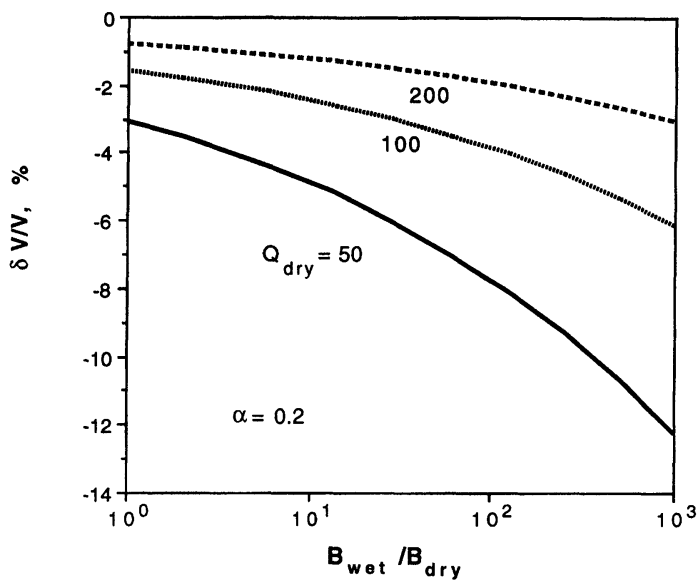

Fig. 1. The effects of water on seismic wave velocities through the enhancement of anelastic relaxation. (A) The ratios $(\delta V / V)_{\text {wet }} /(\delta V / V)_{\text {dry }}$ are plotted against the mobility ratios of defects (such as dislocations) $\mathrm{B}_{\text {wet }} / \mathrm{B}_{\text {dry }}$ for a range of $\alpha$. $\mathrm{B}_{\text {wet }} / \mathrm{B}_{\mathrm{dry}}$ for the upper mantle will vary from $\sim 1$ for dry conditions to $\sim 10^{2}-10^{3}$ for high water fugacity conditions. (B) The velocity reduction due to anelasticity for a range of $Q_{d r y}$ and mobility ratios. For a given $Q_{d r y}$, which is determined by the temperature, $Q$ will be reduced by the presence of water which will enhance the amount of velocity reduction due to anelasticity. The net effects are shown in this diagram as a function of $B_{\text {wet }} / B_{\text {dry }}$ for a range of $Q_{d r y}$. The effects of water to reduce seismic wave velocities can be as large as a few $\%$ for a reasonable range of mobility ratios.

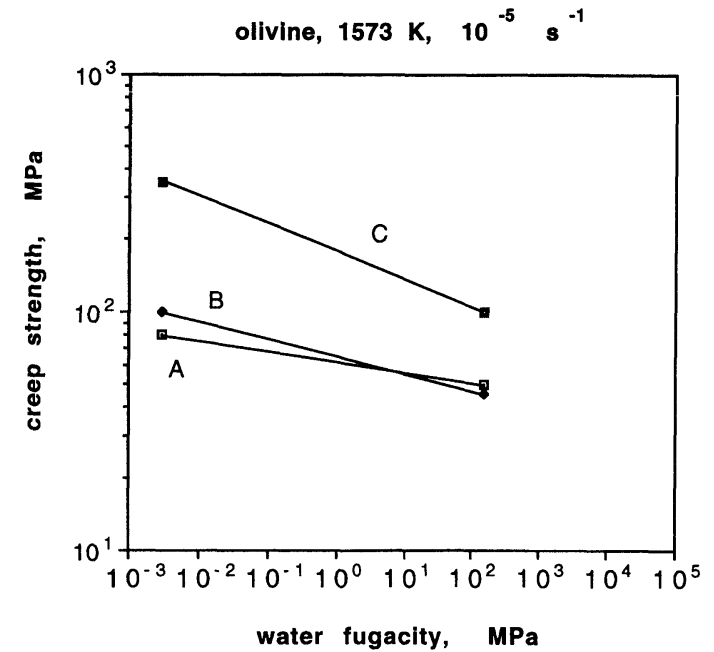

Fig. 2. The effects of water on creep strength of olivine single crystals for various orientations. The data are at $1573 \mathrm{~K}$ and $\dot{\varepsilon}=10^{-5} \mathrm{~s}^{-1}$ ( $\dot{\varepsilon}:$ strain rate) ${ }^{3), 30), 31)} \mathrm{A}:[110]_{\mathrm{c}}$ orientation in which the $[100](010)$ slip system is activated. $B:[101]_{\mathrm{c}}$ orientation in which the $[100](001)$ and the $[001](100)$ slip systems are activated. C: $[011]_{\mathrm{c}}$ orientation in which the [001](010) slip system is activated. Note that the effects of water is larger for the slip systems involving $\mathbf{b}=[001]$ dislocations than for those involving $\mathbf{b}=[100]$ dislocations. The data suggest that the dominant slip direction could change from [100] at low water fugacities to [001] at high water fugacities. in olivine. Note that the effect of water is anisotropic: the effect is large for the $\mathbf{b}=$ [001] (b: Burgers vector of dislocations) dislocations but small for the $\mathbf{b}=[100]$ dislocations. A similar observation was made for dislocation recovery, ${ }^{11)}$ in which the effects of water to enhance dislocation recovery is larger for the $\mathbf{b}=[001]$ (screw) dislocations than the $\mathbf{b}=[100]$ (edge) dislocations. Since the effects of water will be more significant under higher water fugacities (namely under higher pressures), I suggest that creep due to the motion of $\mathbf{b}=$ [001] dislocations might become easier than creep due to $\mathbf{b}=[100]$ dislocations under high water fugacities. Since preferred orientation due to dislocation glide is controlled by the slip systems, such a change in slip direction will result in a drastic change in preferred orientation: olivine [001] axes will be parallel to the macroscopic flow direction under high water fugacity conditions. Fig. 3 compares the seismic anisotropy for the [100](010) slip system under dry conditions with those for other possible slip systems under high water fugacities ([001](100) and [001](010)). It is seen that the seismic anisotropy under high water fugacity conditions will be completely different from that under dry conditions if the dominant slip systems in olivine change to those involving $\mathbf{b}=[001]$. For instance, the polarization of the fast S-waves under high water fugacities will be perpendicular to the flow direction rather than parallel to it. 
(A) water-free

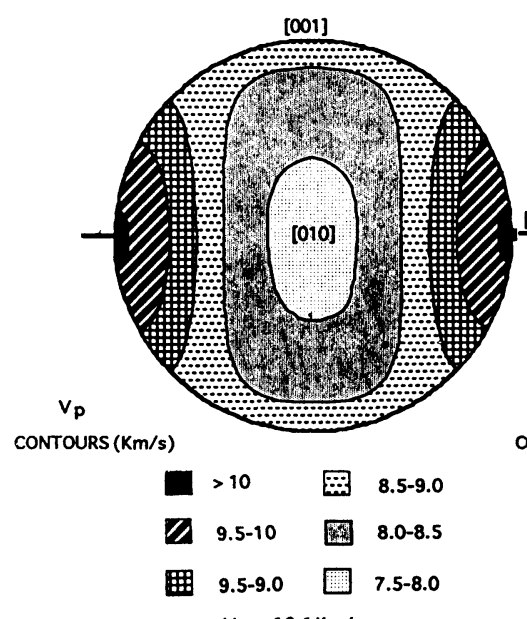

- Max. $10.1 \mathrm{Km} / \mathrm{s}$ Minisotropy $=21.9 \%$

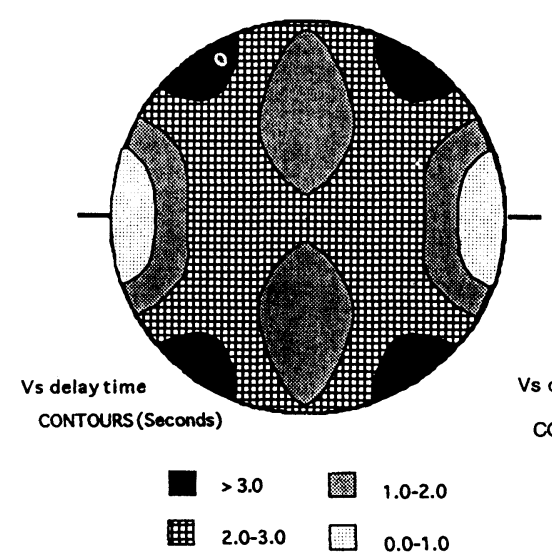

2.0-3.0 Max. $3.5 \mathrm{sec}$. Min. 0. OSec.
Path Lengthe
100.0 Km
(B) water-rich (case I)

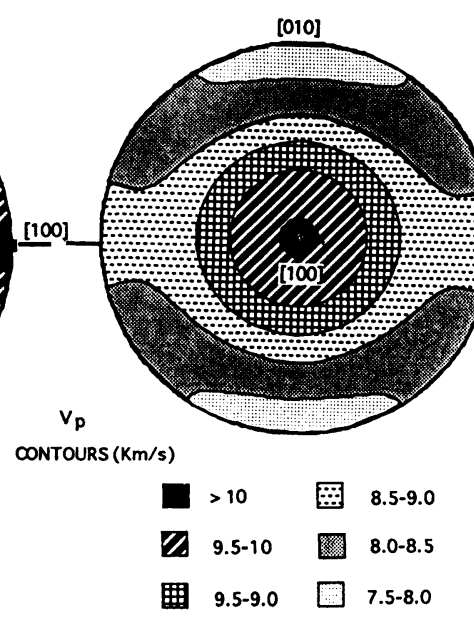

- Max. $10.1 \mathrm{~km} / \mathrm{s}$ Min. $7.9 \mathrm{Km} / \mathrm{s}$
Anisotropy $=21.9 \%$

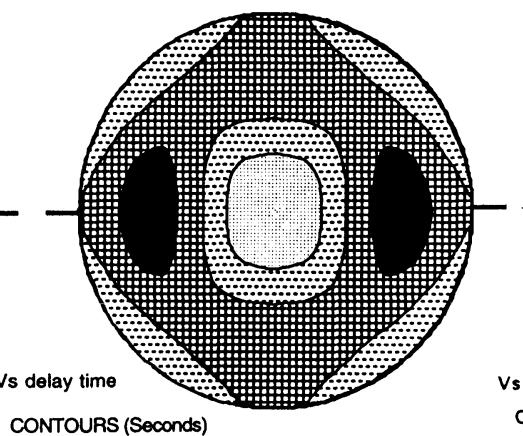

CONTOURS (Seconds)

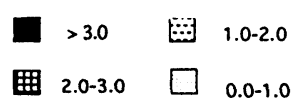

曲 2.0-3.0 $0.0-1.0$ Min. 3.5 Sec. Path Length $=100.0 \mathrm{Km}$
(C) water-rich (case II)

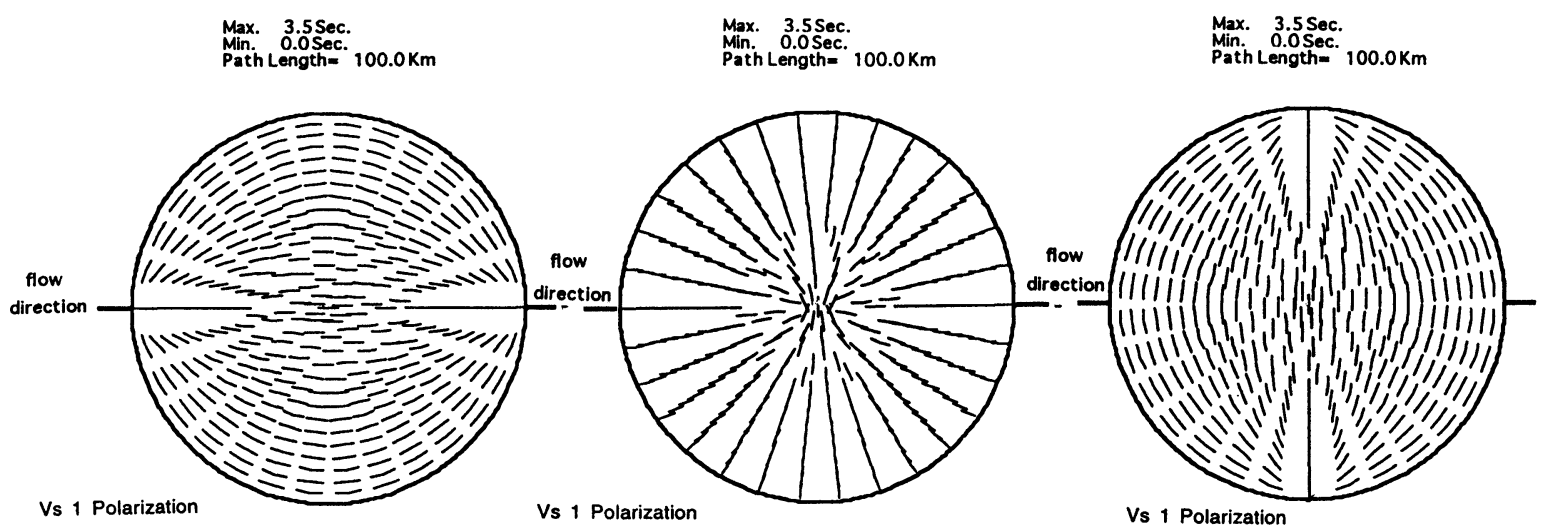

Vs 1 Polarization

Vs 1 Polarization

Fig. 3. Seismic wave velocity anisotropy for different slip systems. Complete alignment corresponding to large strains ${ }^{32)}$ is assumed and hence the results give the maximum anisotropy for a given slip system. In more realistic upper mantle peridotites, the amplitude of anisotropy will be significantly smaller than these values, but the pattern of anisotropy will be similar. Equal area projection is used and the EW direction represents the flow direction. P-wave $\left(V_{\mathrm{P}}\right)$ anisotropy, S-wave $\left(\mathrm{V}_{\mathrm{S}}\right)$ delay time for splitted shear waves corresponding to a $100 \mathrm{~km}$ layer, and the polarization of the faster shear waves $\left(V_{S} 1\right)$ are shown. Under dry conditions (e.g., oceanic upper mantle), the [100](010) slip system dominates and hence the fast P-wave velocity and the polarization of the fast S-wave will be subparallel to the flow direction. When the slip systems with $\mathbf{b}=[001]$ dislocations become dominant, drastic changes in anisotropy would occur: e.g., the polarization of the fast S-wave will be perpendicular to the flow direction. Two cases for water-rich conditions are shown. Case I is the case where the [001](100) slip system dominates and case II is the case where $[001](010)$ slip system dominates. 
Discussions and geophysical implications. The present paper indicates that although the direct effect of water to reduce seismic wave velocities is very small, indirect effects through the change in the mobility of crystalline defects in olivine with water fugacity are likely to be significant. One obvious possibility is the effects through the enhancement of anelastic relaxation and resultant velocity reduction. Although there have not yet been any definitive experimental results to support this notion, a comparison of Sato et al.'s data ${ }^{19)}$ with other data suggests that water supplied by the dehydration of hydrous minerals in Sato et al.'s experiments is likely to have enhanced anelastic relaxation. ${ }^{14)}$ Low seismic wave velocities and high attenuation in back arc basins ${ }^{20)-23)}$ are due presumably to high water fugacities as well as high temperatures. In analyzing these data in terms of temperature profile, ${ }^{24)}$ it is important to take into account of the effect of water separately from that of temperature. Currently available laboratory studies ${ }^{19), 25)}$ did not evaluate the effects of water in detail and the application of these results to the Earth need to be made with caution.

Another mechanism by which seismic wave velocities will be changed by water is the possible change in slip systems in olivine and resultant changes in seismic anisotropy due to high water fugacities. This is admittedly a speculative hypothesis at this moment because no detailed studies have been performed to quantitatively investigate the dependence of creep rate for individual slip system on water fugacity. However, this change in seismic anisotropy provides a possible mechanism to explain the observed two types of anisotropy in back arc basins. Fischer and her co-workers found that the polarization of the fast $\mathrm{S}$-waves is nearly parallel to the trench in some back arc basins with thick crust but it is perpendicular to the trench in others with relatively thin crust. ${ }^{26), 27)}$ One possible mechanism is that the mantle beneath the areas with thick crust is more enriched with water and hence the olivine [001] axis is parallel to the flow direction. In this hypothesis, the observed different orientation of polarization of the fast S-waves does not mean the difference in flow direction. A possible change in preferred orientation is an important hypothesis that can be tested by laboratory experimentation. High pressure deformation experiments with well-defined flow direction (e.g., simple shear deformation) will be needed for this purpose.

Finally, an obvious mechanism of water to change seismic wave velocity which is not discussed in this paper is the one due to the increased amount of melt due to the presence of water. Partial melting will have significant effects on elasticity and anelasticity ${ }^{28)}$ and an increased amount of melt fraction will have an important effect on seismic wave velocities. However, the currently available laboratory data do not provide any definitive conclusions: Sato et al. ${ }^{19)}$ found no appreciable effects of partial melting on seismic wave velocities nor attenuation at high frequencies $(>100$ $\mathrm{kHz}$ ), whereas $\mathrm{Xu}$ and Kohlstedt ${ }^{29)}$ found a significant effect at seismic frequencies $(\sim 1 \mathrm{~Hz})$. The effects are sensitive to melt geometry as well as frequencies and careful studies at seismic frequencies under well controlled melt geometry will be needed to settle the issue.

Acknowledgments. I thank Guust Nolet whose suggestion of an AGU talk on the role of water on seismic wave propagation was the starting point of this study. Thanks are also due to Dave Kohlstedt and Karen Fischer for stimulating discussions. This research is supported by NSF through the grants EAR-9206683 and EAR-9220172.

\section{References}

1) Ringwood, A. E. (1975) Composition and Petrology of the Earth's Mantle. McGraw-Hill, New York, p. 681.

2) Giletti, B. J., and Yund, D. A. (1984) J. Geophys. Res. 89, 4039-4046.

3) Mackwell, S. J., Kohlstedt, D. L., and Paterson, M. S. (1985) J. Geophys. Res. 90, 11319-11333.

4) Karato, S., Paterson, M. S., and Fitz Gerald, J. D. (1986) J. Geophys. Res. 91, 8151-8176.

5) Bell, D. R., and Rossman, G. R. (1992) Science 255 , 1391-1397.

6) Karato, S. (1990) Nature 347, 272-274.

7) Oldenberg, D. W. (1981) Geophys. J. R. astr. Soc. 65 359-394.

8) Bai, Q., and Kohlstedt, D. L. (1992) Nature 357, 672-674.

9) Kohlstedt, D. L., Keppler, H., and Rubie, D. C. (1994) EOS, Trans. Amer. Geophys. Union 75, 652.

10) Karato, S. (1989) Tectonophysics 168, 255-273.

11) Yan, H. (1992) MSc. Thesis, University of Minnesota, p. 98.

12) Watt, P. J., Davies, G. F., and O'Connell, R. J. (1976) Rev. Geophys. Space Phys. 14, 541-563.

13) Karato, S. (1993) Geophys. Res. Lett. 20, 1623-1626.

14) Karato, S., and Spetzler, H. A. (1990) Rev. Geophysics 28 , 399-421.

15) Hess, H. H. (1964) Nature 203, 629-631.

16) Nicolas, A., and Christensen, N. I. (1987) In Composition, Structure and Dynamics of the Lithosphere/Asthenosphere System (eds. Fuchs, K., and Froidevaux, C.). 
Amer. Geophys. Union, Washington, DC., pp. 111-123.

17) Karato, S., and Wu, P. (1993) Science 261, 771-778.

18) Kawasaki, I. (1986) Phys. Earth Planet. Inter. 43, 1-21.

19) Sato, H., Sacks, I. S., and Murase, T. (1989) J. Geophys. Res. 94, 5689-5704.

20) Utsu, T. (1967) Hokkaido Univ., Fac. Sci., J. ser. 7, Geophys. 3, 1-25.

21) Nolet, G., and Zielhuis, A. (1994) J. Geophys. Res. 99, 15813-15820.

22) Zielhuis, A., and Nolet, G. (1994) Science 265, 79-81.

23) Flanagan, M. P., and Wiens, D. A. (1994) J. Geophys. Res. 99, 15469-15485.

24) Sato, H., and Sacks, I. S. (1989) J. Geophys. Res. 94, 5705-5715.

25) Jackson, I., Paterson, M. S., and Fitz Gerald, J. D. (1992) Geophys. J. Int. 108, 517-534.
26) Fischer, K. M., and Yan, X. (1994) Geophys. Res. Lett. 21, 5-8.

27) Fouch, M. J., and Fischer, K. M. (1994) EOS, Trans. Amer. Geophys. Union 75, 450.

28) Shankland, T. J., O'Connell, R. J., and Waff, H. S. (1981) Rev. Geophys. Space Phys. 19, 394-406.

29) Xu, Y., and Kohlstedt, D. L. (1994) EOS, Trans. Amer. Geophys. Union 75, 585.

30) Poumellec, B., and Jaoul, O. (1984) In Deformation of Ceramic Materials (eds. Tressler, R. E., and Bradt, R. C.). Plenum Press, New York, pp. 281-305.

31) Karato, S. (1989) In Rheology of Solids and of the Earth (eds. Karato, S., and Toriumi, M.). Oxford University Press, Oxford, pp. 176-208.

32) Zhang, S., and Karato, S. (1994) (submitted to Nature). 